\title{
The DAM vendor landscape: What the buyer should know
}

\begin{abstract}
Irwin Marcus
Irwin's career has paralleled the rise of digital asset management (DAM) itself, evolving his focus from desktop publishing solutions to departmental production solutions to his current work with enterprise-capable solutions. Currently Irwin has reestablished Marcus Technology after being acquired by Artesia Technologies in May of 2001. During his term at Artesia as a marketing strategist he was responsible for its strategic product marketing focus. His work also included the creation of detailed competitive analysis documents that kept Artesia abreast of their competitors' enhancements and movement in the industry. Irwin's contribution to the industry has been long lasting, molding cutting-edge technology components into solutions for industry-leading clients.
\end{abstract}

Keywords: digital asset management, database, cataloging, object repository, hybrid, file folder, customization, integration, licensing

Abstract There are over 90 different vendors with various types of systems, architecture, and applications. They all have an aggressive sales approach and glitzy marketing information to convey why you should choose them. This paper's main focus will hopefully shed light on the basic DAM categories, an inside/in-depth peek into these categories, the common pitfalls of DAM, and what to look for before deciding on a vendor despite their claims. The majority of research for this paper comes from first-hand experience, user groups and forums, and end-user interviews. To help understand what DAM is all about, a basic DAM primer is included. The importance of evaluating a vendor's Statement of Work (SOW) is outlined in brief here.

\section{INTRODUCTION TO DAM}

Each year, our society generates growing amounts of digital content. This content can include page layouts, high-resolution image files, audio clips, video, marketing collateral, cover art, logos, photographs, legal contracts, etc. Organizing, archiving, and accessing these digital assets across the enterprise can quickly become an overwhelming task.

The basic premise of digital asset management (DAM) is the organization of these digital media files so that authorized individuals can quickly find, retrieve, and physically locate them. It also enables the systematic reuse and reexpression of these digital assets, allowing organizations to stimulate new business models and open up new revenue streams.

Now hundreds of participants including sales, marketing, production, publicists, advertising agencies, and on-demand printers can take advantage of DAM system capabilities.

The next logical next step in the DAM evolution was for vendors to add specialized capabilities to streamline the workflow process by offering a collaborative environment. Now instead of just using DAM as an archiving medium after content had been created, DAM can add value earlier in the creative process. A good example of this is the bridge between the publishing process workflow technology and a DAM system (see, for example, Figure 1).

Another hot area of current DAM development is the method of distributing and updating web content. Automated deployment systems can be used to automate the delivery of the latest product and/or brand image modifications throughout the organization and authorized partners. These automated systems, sometimes referred to as "deployment engines," use business rules to trigger actions. A typical action might be the deployment engine examines asset metadata fields to determine if the asset should be transformed and pushed out to an agency, printer, the web, a portal, e-devices, or simply an FTP site. Logic rules can get quite complicated depending on the audience - see Table 1 for an example. 


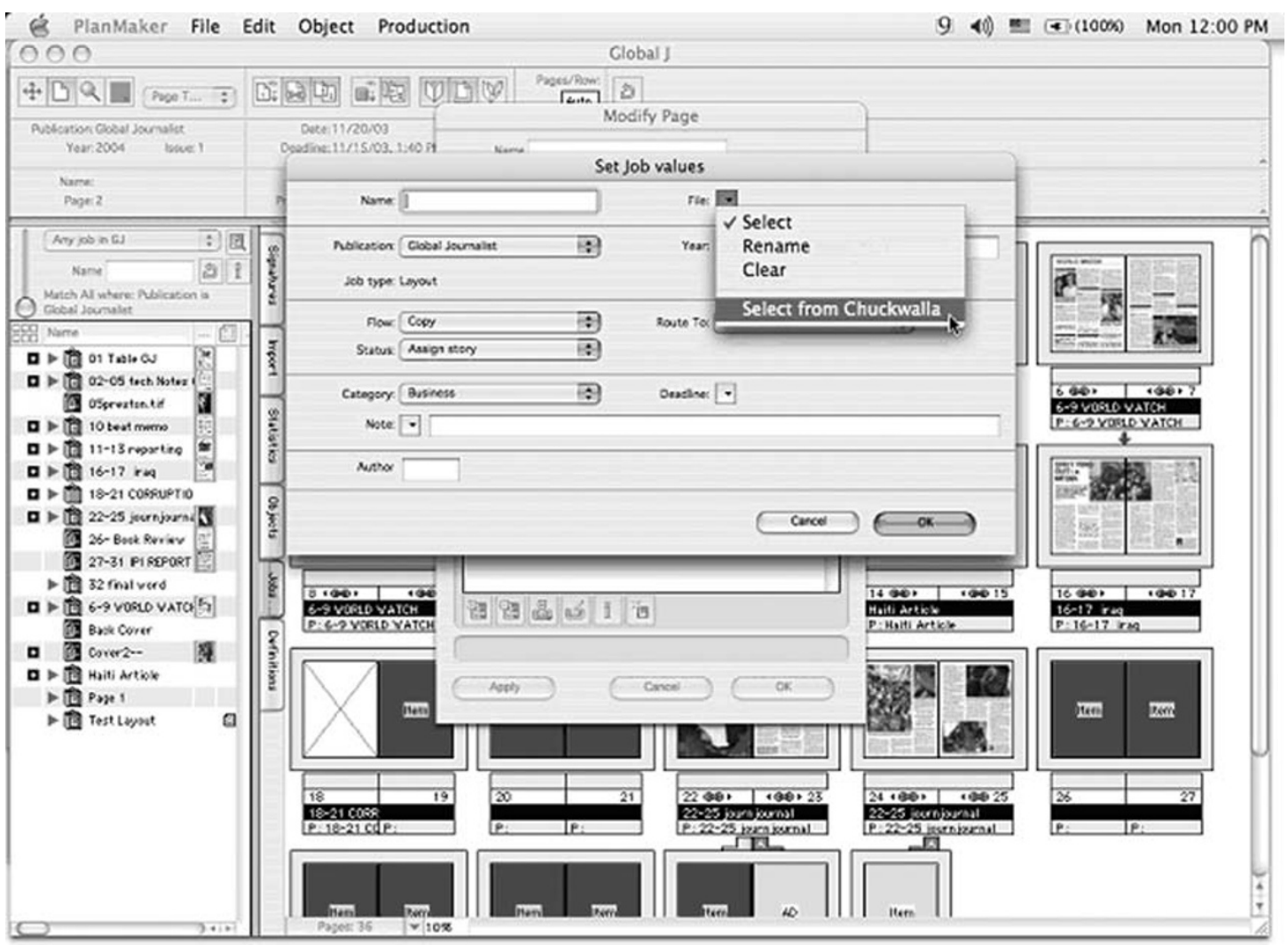

Figure 1: Chuckwalla v5 Integration with Plansystem

\section{DEFINING MEDIA ASSET DATABASES}

Media asset databases can be defined by three different system categories. Each provides unique benefits and trade-offs. The type of system you choose will truly make a difference in the final success of the overall solution.

\section{Are you purchasing a performance workflow tool, distribution system or a digital parking lot?}

The basic DAM categories include cataloging, object repository, and hybrid. Generally, all approaches employ low-resolution thumbnails, a visual browser for viewing (and arranging) these thumbnails, and a search system using metadata and/or keywords. The primary difference of each system type lies in management and security of the high-resolution digital source files.

\section{File folder vs asset orientation}

Another key point of differentiation in the DAM categories is the underlying technology that tracks and controls the asset versus the role the file folder provides.

In the early days before DAM, the folder structure acted as a simple search mechanism for finding and working with jobs. These same folders can now provide a useful abstraction for hierarchical or categorized browsing upon ingestion into the DAM system but that is as far as they go.

Since the essential characteristic of a DAM system is the asset, we should not be concerned with which folder the asset happens to be in. Its role or asset orientation will determine its access level and how we use it.

\section{What is asset orientation?}

At a more technical level, asset orientation implies that security, versioning, transactions, desktop interaction, search results, downloading, etc all rely upon the definition of an asset as the organizing principle. This asset definition includes user-level security — such as roles, levels, and organizations (see Table 2). This is of 
Table 1: Possible "Deployment Agent" decision criteria

\begin{tabular}{|c|c|c|c|c|c|}
\hline \multicolumn{6}{|l|}{ Web } \\
\hline Product & Jpeg & $450 \times 260$ & 72 & Sku-largeview.jpg & TBD \\
\hline Feature Images & Jpeg & $450 \times 350$ & $72-150$ & Filename.jpg & TBD \\
\hline \multicolumn{6}{|l|}{ Majors } \\
\hline Vertical Template & Jpeg & $200 \times 321$ & 72 & Sku-largeview.jpg & TBD \\
\hline Horizontal Template & Jpeg & $400 \times 220$ & 72 & Sku-largeview.jpg & TBD \\
\hline Feature Images & Jpeg & $450 \times 350$ & $72-150$ & Filename.jpg & TBD \\
\hline \multicolumn{6}{|l|}{ Countertop } \\
\hline Vertical & Jpeg & MAX:257 × 263 & 72 & Sku-largeview.jpg & TBD \\
\hline Horizontal & Jpeg & MAX;500 × 200 & 72 & Sku-largeview.jpg & TBD \\
\hline Images for Rollover* & Jpeg & Max:85 × 100 & 72 & Sku-smallview.jpg & TBD \\
\hline \multicolumn{6}{|l|}{ Appliance 1} \\
\hline Store A & Jpeg & $1000 \times 1000$ & 150 & UPC code.jpg & TBD \\
\hline Store B & Jpeg & Same as $A$ & 72 & Sku-largeview.jpg & TBD \\
\hline Store C & Eps./Tiff & Same as $A$ & 72 & Sku.eps & TBD \\
\hline Store D & Jpeg & $5 \times 7$ inches & 300 & Sku-largeview.jpg & TBD \\
\hline Photo (guide) & Tiff & $3.75 \times 5$ inches & 250 & Sku.tif & Internal server \\
\hline Photo (default jpg) & jpeg & $5 \times 7$ inches & 150 & File name.jpg & Internal server \\
\hline
\end{tabular}

Note: These rules will be strictly administrative access, not available as pop-up choices to general users

Thus, IF (Query metadata fields)

If Brand="XXX" or "YYY" or "ZZZ" AND

If setting type description="outline" AND

If product status = "production" AND

If push status = "Needs to be pushed"

THEN

Transform object based on matrix (see matrix below), rename asset based on matrix and export to destination specified by matrix.

1. If Brand $=x$, transform to Target Format $x$, rename if needed, and export

2. If Brand $=y$, transform to Target Format $y$, rename if needed and export

3. Reset push status to "sent" or something to indicate it got sent already and not to do it again

critical importance for many corporate group requirements.

A digital asset can be simply stated as:

content + metadata $=$ asset

Metadata can include information about format type, rights and permissions, usage history, keywords, etc. This kind of information is typically well-suited to be stored in fields such as simple data elements and optimized index tables.

Other types of related information are more appropriately modeled as relationships between assets. For example, when the rights and permissions that govern the use of a digital asset are extremely complex, a link to the original contract might be required - these links are supplements to the classic metadata fields.

\section{THE DAM CATEGORIES}

\section{Cataloging}

Cataloging systems such as Canto "Cumulus," Extensis "Portfolio" or Wave "Media Bank" capture only the low-resolution thumbnails, descriptive metadata and a pointer to the location of the source media asset; this process leaves the source files intact, residing in the original job folders from which they were cataloged. The search facility enables a user to quickly browse a collection of images and retrieve the full resolution media file directly from its place on the fileserver.

An advantage of the cataloging system is that it should interact well with others, ie it should be open and easy for other software applications to access the same stored digital assets controlled by the DAM system.

The purpose of the Open Press Interface (OPI) for example, is that it allows designers to build layouts with low-resolution proxies. When the layout is output to an OPI-enabled print queue, the OPI server replaces the lowerresolution proxy image with its high-resolution counterpart on the fly. OPI software must have open access to the high-resolution files that reside on the file server. The cataloging systems have the asset path and file system openly available, whereas the asset repository does not. 
Table 2: Artesia's role-based security

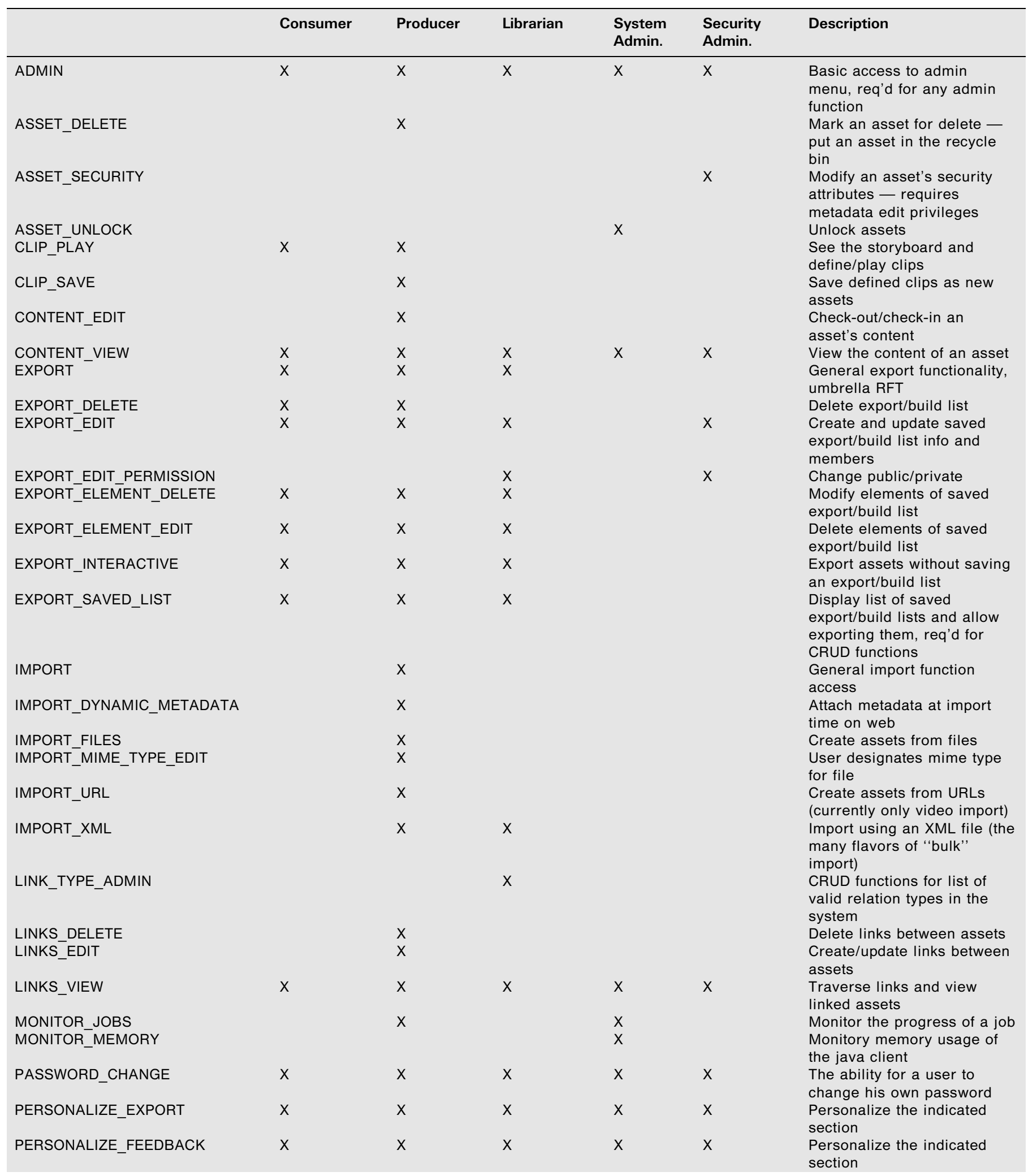


Table 2: Continued

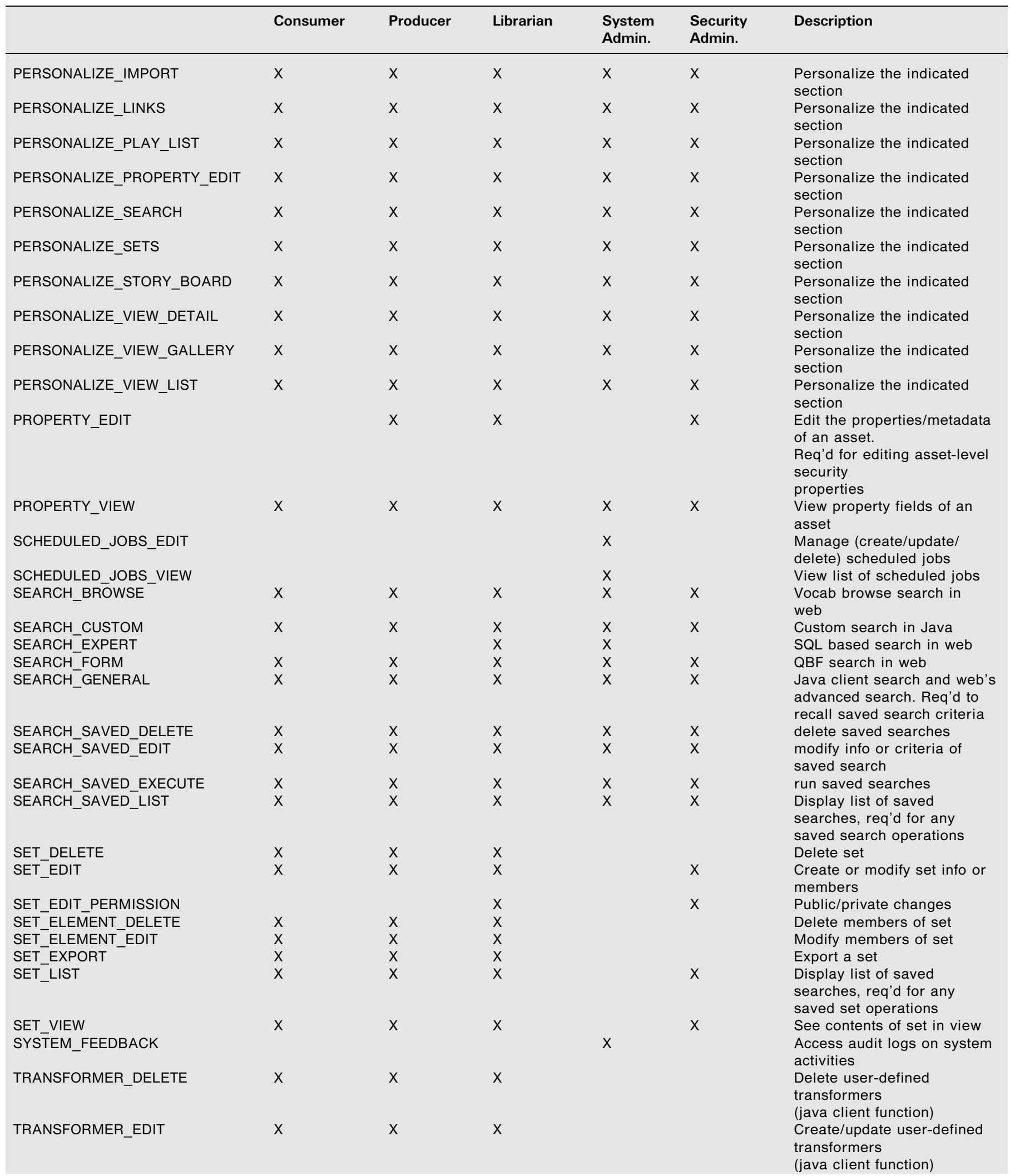


Table 2: Continued

\begin{tabular}{|c|c|c|c|c|c|c|}
\hline & Consumer & Producer & Librarian & $\begin{array}{l}\text { System } \\
\text { Admin. }\end{array}$ & $\begin{array}{l}\text { Security } \\
\text { Admin. }\end{array}$ & Description \\
\hline TRANSFORMER_VIEW & $x$ & $x$ & $x$ & & & $\begin{array}{l}\text { View and apply transformers } \\
\text { to assets during export }\end{array}$ \\
\hline TRANSFORMER_ADMIN & & & $x$ & & & $\begin{array}{l}\text { Run the transformer admin. } \\
\text { utility }\end{array}$ \\
\hline USER_ACTIVE_LOGIN & & & & $x$ & & $\begin{array}{l}\text { View list of users currently } \\
\text { logged into the system }\end{array}$ \\
\hline USER_FEEDBACK & $x$ & $x$ & $x$ & $x$ & $x$ & $\begin{array}{l}\text { Ability to view logged } \\
\text { information about } \\
\text { user-submitted jobs }\end{array}$ \\
\hline VOCAB_ADMIN_EDIT & & & $\mathrm{x}$ & & & Modify thesauri \\
\hline
\end{tabular}

Note: The chart depicts the granularity of rights that can be given for a particular group. Each user is assigned a role based upon the user's area of responsibility within the organization

Another discussion point with cataloging systems is they are intermediate in cost, scalability, and ease of use. They are also vulnerable to end users possibly assessing the digital assets directly from the file-server, working "behind the system's back" thereby introducing human error and negating the control and stamping of the digital assets by the DAM system.

\section{The managed file folder}

In the file folder system, such as "XiNet" or "MediaBeacon," the web interface browses and directly retrieves assets from the file system. The database provides a web front-end, but acts just like a user opening a server share - the front end becomes just another concurrent form of access to the file system.

Many of these systems cannot check-in or check-out assets because there is no way of controlling the outside environment. They are weak at preventing users from getting to assets that should have limited access or might be locked for an update process. The file system is the weak link and the security for managing viewing, extracting, and updating file folders is a manual process that can become a laborintensive job depending on the complexity of the viewing community.

\section{Managed file folder security}

With folder level security, such as Interwoven "Media Bin," if there is a need to filter access of assets to a specific group, it must be done manually. ${ }^{1-4}$ You must actually move and place the specific assets into the appropriate folders that have the correct rights associated with them.

These systems are easier to administer but more manually intensive to control how the assets are available for access.

\section{Object repositories}

Object repositories such as Artesia's “Teams" capture not only the thumbnails and metadata but also the high-resolution asset itself. The thumbnails and metadata become part of the centralized database and at the same time the high-resolution assets are moved from the file system into their specially accessed vault-like data warehouse, thereby removing the file from the user's standard job folder structure. Since the asset is in a secure encrypted folder, it is not accessible from the desktop or other third party software products (such as OPI) without specific application programmming interface (API) programming. The asset path and its access can only be found and retrieved by the database.

This approach is robust, secure, and scales up for very large organizations, but it is also very expensive. And it is not entirely foolproof; assets still have to be copied to a file system (via a check-out procedure) before they can be modified, leading to multiple unmanaged copies of an asset.

\section{Hybrid systems}

Hybrid systems such as North Plains'

"Telescope" or "Flexdb" (an open source product) do not require the proprietary ingestion of digital files in order to manage them as with the object repositories. They allow all the assets to appear in one central virtual repository and 
use either peer-to-peer processing or file brokers to retrieve from multiple file-servers without the need to change network and storage infrastructure or MIS practices.

The file brokers or special database services (new in Canto "Cumulus 6") let creator applications (eg Quark XPress or Adobe Photoshop) read and write files that are native to the host operating system (eg MacOS), yet the servers always store files in their native formats. Brokers act as network drivers (acting at the application level, not as part of the operating system) so they are essentially invisible to the user.

\section{Hybrid combination systems}

A hybrid combination system, such as "Chuckwalla v5," can be set up as both an object repository and/or cataloging system, depending on best practices for your usage and environment. This simply means that Chuckwalla allows concurrent support of either "cataloging" or "ingesting" elements into the repository on an individual asset-by-asset basis. This offers tremendous benefits depending on security concerns, openness, simplicity of an external third party application access of stored assets, speed, etc.

\section{THE MACINTOSH}

If Macintosh functionality is important to you, the first question you must ask yourself is whether the machines are going to be used in production or just for web browsing and downloading? If the latter, then your problems are pretty well minimized and most systems will support this functionality. If the answer is in production and your creative people need to build Adobe In-Design or Quark documents, then typically you need a specialty "fat" client or a "smart" client.

A smart client operates from within the design or desktop application and has a plug-in which can query and directly retrieve (drag and drop) assets from the database for the building of pages.

A fat client is a specially designed application that takes advantage of the PC or Macintosh operating system to enhance workflow such as building pages, etc. For example QuarkDMS, "Chuckwalla," and "Telescope" all have special features for QuarkXPress, which include the deconstruction of a Quark document. This enables each component of a Quark document to be added to the database as an asset which greatly enhances the workflow process.

"Chuckwalla v5" and "Telescope" have recently added this same compound document deconstruction support for Adobe In-Design.

A problem in working with a fat client and/or web client is that they must support the older Macintosh files (ie anything before OSX). The systems had to deal with both a "data" and "resource" fork. Many systems did not handle this well - especially repositories. The new OSX operating system combines both the data fork and resource fork into one file, thereby simplifying the DAM system design.

There are a number of other things to look out for when integrating the Macintosh. One issue depends on if you are running on Sun Solaris or Windows 2000/NT as your file server. Does the DAM system demand you to run unwanted or unsupported protocols on your local area network (LAN)? For example one system may demand the use of the Mac Network File System (NFS) protocol running on your LAN to support the access of these files. This protocol would cause issues running across the internet as well as possible conflict with your internal network operations.

Another issue and potential drawback in a system design is if the system must download all high resolution embedded files to a temporary server share before a program such as Quark can print them, or before they can be exported and transformed into another type of asset. This translates to major delays in system performance. For example if you have $1 \mathrm{~Gb}$ of assets placed in a Quark document, the entire 1 Gb's worth of data must be passed to a temporary mounted file system, then the same $1 \mathrm{~Gb}$ 's worth of data would be sent to the RIP, printer, or imposition software. This approach in system design makes it difficult to be used in a fast-paced production environment.

Also be aware that some systems might not be able to handle both the OSX and pre-OSX operating system platforms.

\section{EASE OF USE AND ADOPTABILITY}

\section{Tagging data}

With digital content, all of the content's metadata - the information describing each image, audio file or video - have to be created. 
Before assigning metadata - which is really data about data - you will need to build a clear model and consistent format for every type of digital asset. Use whatever descriptors are needed to cover the logical means of identifying each a digital asset — but not so much that retrieval becomes unwieldy.

Most systems have an automated batch method of assigning metadata upon import. Theses systems use the concept of hot "import" folders. The administrator can assign templatebased metadata that are automatically assigned to each asset that is dropped into the appropriate hot import folder.

One system such as North Plains' "TelescopeImport Wizard" has a more advanced intelligent business logic, which can extract information from the file structure automatically. The folder and sub folder names are read and extracted using a rule-based scripting language and stringmanipulation functions. This can be extremely useful in saving time and intensive manual labor.

\section{DAM not an extra step}

To avoid owning a "digital parking lot" you must have a system that functions efficiently and minimizes extra steps. Creative people will resist and perhaps even avoid new processes that add extra time to their work process and/or another application sharing their desktop. To that end, some vendors have made their tools integrate with creation software such as Quark XPress and Adobe In-Design.

"Portfolio Server" from Extensis lets users access images and graphics directly from within design tools such as Adobe Photoshop and Quark XPress. Another DAM vendor, INSCI

(Webware) has released their ActiveMedia

Desktop Client Connection. This plug-in functions similarly to the "Portfolio Server" by allowing Macintosh or PC workstations to access the DAM repository through Quark XPress or Adobe In-Design. A typical Quark or In-Design layout file may include dozens of linked files, such as graphics and photos. The ActiveMedia plug-in lets users save their new designs into Insci's central repository, and it also lets users place files from the repository directly into their layout.

\section{THE VENDORS}

Companies need to choose a DAM vendor from an informed position, and to protect themselves from being exploited before signing an agreement. Once the system is purchased and implemented, it is almost impossible to back out. Even though the vendor speaks DAM-ese and boasts about a feature or two, that does not necessarily translate into an optimal system.

There is a case in which a client was dissatisfied with a vendor's implementation. Although the system did function, it did not operate to the capability that was sold and would only minimally meet the organization's needs. Unfortunately there were a number of important items that were overlooked in the pre-sales analysis of the software application. When the problems turned out to be very real roadblocks in an optimized solution, the client now had a choice of working around the issues or paying the vendor thousands of dollars for a customized solution extension.

Since the client had invested millions of dollars in the software, human resources, and the infrastructure needed, the vendor knew that it would be next to impossible for their client's management to secure funding to replace the system and would not want to get involved in a costly legal battle. The client wound up keeping the solution and spending many hours on a continuous basis working around the shortcomings of the software.

Thus, the relationship became and continues to be strained between the vendor and client the users and management have a poor experience with the software application and the company that supports the application - this type of situation can be avoided with the proper business requirements analysis and due diligence.

\section{Honesty and integrity}

The above example leads us to an item that is perhaps more important than the technology itself - the honesty and integrity from which the vendor operates. You must get to know the vendor - speak with their clients. Try to speak to clients that are not on their approved reference list. The software run by your customers or peers is usually common knowledge. Do a little digging and speak to these users directly, not through a vendor conference call.

- Try to ascertain if the vendors were willing to make changes in software based on trends in the industry. How long do they take? 
- Were they sensitive to client needs or extremely slow at adopting the customer requests as enhancements for future releases?

- How well did their deployment strategy and subsequently their implementation work?

- How long did it take for the users to adopt the system?

The success of the vendor/client relationship is not just the coordination of the integration and support personnel that would help avoid hazards — the vendor management must set up the internal mechanisms to foster a truly innovative aggressive policy.

\section{Statement of Work}

Once the vendor of choice is made and you are satisfied with their technology and the technical details such as solution architecture, equipment, workflow, business metrics, and training is worked out - create or get a detailed Statement of Work (SOW) tied to your agreement. Make sure it also includes performance criteria of the system, expected completion dates, and an exit strategy if those conditions are not met.

Never be rushed to make a decision - too often the vendor tells you they are anxious to get the deal signed before the end of the quarter or they can substantially discount the system if they could place your order by the 30th. This can lead to disastrous consequences if you move too quickly and accept verbal commitments.

Once you issue a purchase order and the check is in the vendor's hands, you are at their mercy to live up to any verbal agreements you discussed.

We are not saying that all vendors are slippery - but for business' sake, it is better to secure and sign all paperwork such as the purchase, support-maintenance agreement, and SOW at the same time.

\section{Hidden expense: Customization and integration}

\section{Customization}

Vendors are sometimes reluctant to be truthful about the degree of customization and integration that is required of their products.

Some work hard to hide these facts because they derive a major portion of their income from these customizations. Unfortunately, clients often find out after the sale that a common function that they saw demonstrated in a presales meeting turns out to be a costly customization.

For example, in a number of cases, potential customers observed a Macintosh fat client demonstrated with full support for the Macintosh file (data fork and resource fork) but after the system was deployed they were dismayed to find that the resource fork was lost over the internet when they utilized the web browser. Now the customer was faced with costly customizations or they had to manually recreate the resource fork information every time they downloaded an asset over the internet.

\section{Productizing}

A number of vendors do not disclose the fact that they have already created a popular feature/ customization for another customer. Rather than productizing a popular request from their customers, they will charge the full custom development fee to another customer showing interest in the same type of features.

A potential solution for solving this dilemma would be to join a user group. Clients can learn from each other's experiences and discuss common shortcomings of the software. In one group I know, some customers found they all had similar customizations and code for which they all paid a premium.

\section{Warrantee}

Many vendors do not support or warrantee their customizations. This creates a huge problem when moving forward if your organization does not have the technical expertise to maintain the product. For example, one vendor calls their customization a "solution accelerator." This "accelerator software deliverable" is a professional service engagement that sells for a fixed fee under a work order. It is not covered under standard support and maintenance. There are no upgrade commitments and no warranties. It is a customization which the customer is expected to maintain and support.

\section{Customization and the dot upgrade}

New vendor software releases will not include the customer's customizations and in fact might break the code when the upgrade is put in place. The customer must pay the vendor to 
recondition the code to work with the new release.

One way to minimize this issue is to create the customizations using the vendor's standard API interface. Usually this interface does not change from version to version.

\section{Customization and documentation}

If a vendor customization is part of your SOW make sure the vendors are required to document their work and request this as soon as the customization is completed.

In one example, a client never received the proper documentation. The client used an internal IT person who took eight weeks to document all the code delivered to them. They also found that they had been misled about the complexity of the custom code that interfaced with the application's services. This one facet itself made it extremely costly for the client to upgrade to a new release of core software because of all the changes necessary to make the custom code operate with it.

\section{ADMINISTRATION OR CUSTOMIZATION}

In different ways and different proportions, each vendor's solution encompasses three basic elements - application, platform, and integration.

The most costly and time-consuming solution is one that depends on custom development for the majority of its functionality and use. The only time that this type of system can be justified is by a set of requirements that are both substantially unique and promise to fill a mission critical function for the sponsors of the enterprise. We all know that highly skilled labor is hard to come by and expensive.

For example, the non-administration "gotcha." You want to make searching easier for users to find a new brand name product, so you decide to add a pop-up listing in the product's metadata field. In one system the task of adding this pop-up label would be an administration function, in another system it would be a complex customization using complex structured query language (SQL) query code.

Since all systems require ongoing configuration and administration, it is best to choose a system that is substantially driven by an underlying application. As such, the sophistication needed to handle simple changes is drastically less.

Some system solutions are considered shrinkwrapped and/or turnkey. These systems are the most desirable and simplest to justify and adopt but may be limited in functionality - the idea is to find the solution that offers both.

\section{SOFTWARE LICENSING}

\section{CPU-based}

For medium to large-scale implementation vendors usually charge a central processing unit (CPU)-based fee. This fee is determined by the quantity of CPUs running the vendor's application on one or more servers. Usually additional processors are discounted from the single CPU list. Usually the processor size/speed and number of users per CPU is not a factor in determining the pricing.

Some vendors charge separately for their local client applications vs their web browser interface. In other words they can charge a CPU-based fee and then additionally charge for web access.

\section{Named licensing vs concurrent}

To help lower the cost in the early stages of a DAM implementation, vendors usually offer various limited license packages.

With named licensing they charge a fee for each individual user that needs to access the system. This type of licensing can be very annoying to administer and is not free flowing. For instance the individual license might be tagged to the application running on an individual machine. This would be problematic if a user moves to different locations or different machines.

With concurrent licensing the vendor application can be installed on any number of computers and will reach its limit when the number of users logged in at that time exceeds the limit count. This type of license is preferred for the type of company that has two or three shifts. If there are not more than 20 users per shift, then the concurrent license could be limited to 20, since in the named licensing you would have to purchase 60 licenses.

To determine which license is appropriate for your company you should ascertain: 
- What is the cost difference per seat or per server CPU?

- If it is based on the server - do they take into account CPU speed, number of processors, etc?

- What is the cost of additional processors?

- Is the web browser client licensed the same as a specific PC or Macintosh client?

- Do they charge for a software developer's kit (SDK) - developer license? Is an SDK available?

- Do they charge for administration applications or licenses?

\section{INTELLECTUAL PROPERTY OWNERSHIP INVOLVING CUSTOMIZATIONS}

When you purchase a software license from a proprietary DAM vendor you will not have access to the underlying source code. Usually vendors do have APIs, Java applets, or can supply a database schema that will enable you to control and exchange information to and from the DAM system using their SDK.

For customizations, it is another story. When customizations are necessary you should retain ownership of the source code that is created for you. You also want to make sure that the software is properly documented and turned over when complete.

\section{SOFTWARE LICENSING AND HIDDEN CHARGES}

Some vendors levy a connectivity fee if you use an external application which accesses assets in your own database. The vendor rationale is that you created the database schema with their software, therefore they can levy a charge at their discretion.

An example of an external application would be your own web interface extracting metadata, thumbnails or assets, or even just Microsoft Excel connecting to the database to extract client usage information.

The paragraph below is an actual excerpt from a major DAM vendor's end-users' license agreement. Please note there is no cost listed for the supplemental fee of $(\mathrm{x})$ times the vendor variable (y) for any amount of time ( $\mathrm{t}$ ). I would not think a legitimate business could get away with this type of open-ended stipulation.

CONNECTIVITY FEES: End User acknowledges that it may not connect to a XX database using any other software product, other than the Software, without first paying Vendor a supplementary database connectivity fee. In such case End User agrees to pay to Vendor such database connectivity fee as set in place by Vendor from time to time.

\section{AN ALTERNATIVE TO THE STANDARD VENDORS: OPEN SOURCE}

Open source software/free software (OSS/FS) has risen to great prominence and is a model used by developers for years. It has served as the foundation for many of technology's great advances, including the internet, world wide web, Linux Operating System, and the Apache HTTP Web server. Although there are variations in licensing, the principal concept is to promote collaboration among developers to create the most viable and cost-effective solution for a specific community or organization. This collaborative model allows organizations to reuse, extend, enhance, and leverage community skills, which ultimately ensures the preservation of information systems and protects existing investments in business applications.

It is now possible to add capability to an existing DAM system with a variety of open source applications. Another possibility is to replace a DAM system with an open source enterprise-class, digital asset management solution. There are core DAM systems now available in the public domain that are built upon an extensible, object-based architecture.

There are still many questions to be asked and answered before moving to an open source solution such as:

- What is the true cost saving of migrating to open source?

- What are the largest risks associated with open source software and how can you most effectively mitigate those risks?

- Who will support the system?

- What are software development costs?

- When is the right time and situation for your organization to use or migrate to an open source platform?

To help substantiate and direct the open source movement with experience in the DAM space there are two initiatives. 
1. Project Avalanche. This is a legally constituted intellectual-property cooperative. Companies pay $\$ 30,000$ a year to become members. They can then donate any in-house software they choose to the Avalanche library, with the project becoming the legal owner of the code. Project members get to use, free of charge, any of the other programs in the library. Avalanche already has a number of impressive sponsors, including names like Best Buy, Jostens Inc., Cargill, Medtronic, and Integral Business Solutions who built some of its components.

2. The Open Advantage Coalition (OAC). This coalition, recently created by the Chalex Corporation and the Fort Dearborn Company (as a major sponsor), is made up of developers and commercial investors and dedicates itself to the development, integration, and implementation of open source solutions that support the print, publication, and graphic arts industries.

\section{The effectiveness of open source}

Eric Raymond's essay, The Cathedral and the Bazaar, presents a compelling argument on the effectiveness of open source software projects; Raymond defines 19 points to guide the administration of an open source project in a nurturing way. Users of open source software also have access to its source code, and are encouraged to participate in its constant improvement.

While coding remains an essentially solitary activity, the really great hacks come from harnessing the attention and brainpower of entire communities. The developer who uses only his or her own brain in a closed project is going to fall behind the developer who knows how to create an open, evolutionary context in which feedback, exploring the design space, code contributions, bug-spotting, and other improvements come from hundreds (perhaps thousands) of people. ${ }^{5}$

\section{OVERCOMING ISSUES IN ADVANCE}

\section{Testing and benchmarking the system} It is important to test and try and expose every facet of the system, how you intend to use the system, and your expected results. Supplying test files that are the actual size files that you are working with is important. Don't fall for the line that the vendor does not have the manpower or servers available to test your type of file. Many times vendors strip down files, remove elements and/or alter the resolution of the image to make their system seem faster.

\section{Speed vs cost for license and hardware fees}

When a DAM vendor's system is slow and nonresponsive, multiple hardware CPUs and memory must be purchased for the system to function properly. This becomes an escalating expense since you now also have to pay the vendor their multiple CPU license fee. As shown in this model, the vendors do not have the pressure on themselves to make their systems more efficient and faster. They actually make more money on a system that is inherently slower.

\section{Other questions}

- Can the system use open source components such as the database, web server, etc?

- Are the service components server side or client based? Remember in certain cases local client services may perform as well as server based

- Does the system have high availability architecture? (eg fault tolerance, fail-over, service replication, load balancing)

- Compliance to industry standards?

\section{CONCLUSION}

Today's vendors need to provide solutions that are continually evolving. The exchange, sharing of ideas, and trust of your vendor is most important.

A sure way to be successful is to learn from other successful organizations. We cannot stress enough that you should talk to real users. Ask the vendor for the user group list. Make calls to people you might know.

Remember an effective DAM solution will be focused on dual goals: comfortably fitting into the normal work processes of end-users, and supporting how the enterprise produces and delivers content through a variety of channels. 


\section{APPENDIX}

Vendors and/or projects mentioned in this paper:

Artesia Technologies

http://www.artesia.com

Avalanche Corporate

http://ibsmmap002.go-integral.net

Brighttech Inc.

http://www.mediabeacon.com

Canto Software Inc.

http://www.canto.com

Chalex Corporation

http://chalexopenadvantage.org/index.html

Chuckwalla Inc.

http://www.chuckwalla.com

Extensis Corp

http://www.extensis.com

FlexStor Corp

http://www.flexstor.biz

FlexStor - Open Source

http://www.flexstor.org

Insi - Webware

http://www.insci.com

Integral Business

http://www.go-integral.com
Interwoven Inc.

http://www.mediabin.com

North Plains Inc

http://www.northplains.com

Quark Inc.

http://www.quark.com/products/quarkdms

Wave Corporation

http://www.wavecorp.com

\section{References}

1 Gotcha! Utilizing Digital Asset Management http:// www.baselinemag.com/article2/

0,1397,886786,00.asp

2 What Is a Digital Asset? http://

www.baselinemag.com/article2/

0,1397,886897,00.asp

3 Digital Asset Management Goes Enterprisewide http://www.transformmag.com/contentmgmt/ show Article.jhtml?articleID = 15100002\&pgno $=2$

4 Is Asset Management a Business? http:// www.dpsmagazine.com/Features/F_2-2/ assetmgmntAH.htm

5 Raymond, E. S. (2001) The Cathedral and the Bazaar: Musings on Linux and Open Source by an Accidental Revolutionary, 2nd edn, O'Reilly, UK. 